\title{
ERRATUM
}

\section{Novel adeno-associated viral vectors for retinal gene therapy}

\author{
LH Vandenberghe and A Auricchio
}

Gene Therapy (2012) 19, 236; doi:10.1038/gt.2011.199; published online 24 November 2011 Correction to: Gene Therapy (2012) 19, 162-168; doi:10.1038/
gt.2011.151; published online 13 October 2011

Since the online publication of this paper the authors have noticed that the sixth sentence of the second paragraph of the Conclusions and Prospects section was incorrectly changed by the copy editor. The correct sentence is shown below:

Ultimately, one can envisage a scenario in which extensive efficacy testing in small animal models will no longer be required: one may develop a therapeutic AAV vector based on the most efficient serotype selected in NHP or human retinal culture, test its potency (that is, ability to express the therapeutic transgene) in explants and directly progress to production of vector under Good Manufacturing Practice standards and assessment of safety and toxicity before going into humans.

The copy editor would like to apologize for this error. 